CERN-TH.6628/92

CTP-TAMU-61/92

ACT-19/92

\title{
Proton Decay and Cosmology Strongly Constrain the Minimal SU(5) Supergravity Model
}

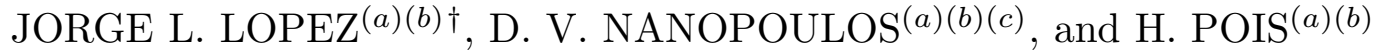 \\ (a) Center for Theoretical Physics, Department of Physics, Texas A\&M University \\ College Station, TX 77843-4242, USA \\ (b) Astroparticle Physics Group, Houston Advanced Research Center (HARC) \\ The Woodlands, TX 7r381, USA \\ ${ }^{(c)}$ CERN Theory Division, 1211 Geneva 23, Switzerland
}

\begin{abstract}
We present the results of an extensive exploration of the five-dimensional parameter space of the minimal $S U(5)$ supergravity model, including the constraints of a long enough proton lifetime $\left(\tau_{p}>1 \times 10^{32} \mathrm{y}\right)$ and a small enough neutralino cosmological relic density $\left(\Omega_{\chi} h_{0}^{2} \leq 1\right)$. We find that the combined effect of these two constraints is quite severe, although still leaving a small region of parameter space with $m_{\tilde{g}, \tilde{q}}<1 \mathrm{TeV}$. The allowed values of the proton lifetime extend up to $\tau_{p} \approx 1 \times 10^{33} \mathrm{y}$ and should be fully explored by the SuperKamiokande experiment. The proton lifetime cut also entails the following mass correlations and bounds: $m_{h} \lesssim 100 \mathrm{GeV}, m_{\chi} \approx \frac{1}{2} m_{\chi_{2}^{0}} \approx 0.15 m_{\tilde{g}}, m_{\chi_{2}^{0}} \approx m_{\chi_{1}^{+}}$, and $m_{\chi}<85(115) \mathrm{GeV}, m_{\chi_{2}^{0}, \chi_{1}^{+}}<165(225) \mathrm{GeV}$ for $\alpha_{3}=0.113$ (0.120). Finally, the combined proton decay and cosmology constraints predict that if $m_{h} \gtrsim 75(80) \mathrm{GeV}$ then $m_{\chi_{1}^{+}} \lesssim 90(110) \mathrm{GeV}$ for $\alpha_{3}=0.113(0.120)$. Thus, if this model is correct, at least one of these particles will likely be observed at LEPII.

CERN-TH.6628/92

CTP-TAMU-61/92

ACT-19/92
\end{abstract}

August, 1992

$\dagger$ A condensed version of this paper will appear in the Proceedings of the XXVI International Conference on High Energy Physics, Dallas-Texas, August 5-12, 1992. Conference Speaker. 


\section{Introduction}

The minimal $S U(5)$ supergravity model [i] has recently passed the simplest possible consistency check, namely the unification of the gauge couplings at an energy scale $M_{U} \sim$ $10^{16} \mathrm{GeV}$ [2]. This check depends only in sub-leading order on the masses of the light and heavy particles in the theory, and as such provides weak constraints on the various model parameters [3]. On the other hand, the requirement that the dimension-five-induced proton decay operators [4] be within current experimental bounds provide rather stringent constraints on all sectors of the theory [5, 6, 6, 8, 8, 9, 10]. Recently two of us (JL and DVN) with A. Zichichi [11], studied a representative set of points in parameter space which satify the proton decay bound and applied to these the cosmological requirement $\Omega_{\chi} h_{0}^{2} \leq 1$, where $\Omega_{\chi}$ is the relic abundance of the lightest neutralino (also the lightest supersymmetric particle (LSP) and which is assumed to be stable) and $0.5 \leq h_{0} \leq 1$ is the Hubble parameter. We found that the cosmological constraint was grossly violated for these points. It was also noted that there may still exist cosmologically allowed regions for sufficiently small values of $m_{t}$. In this paper we present a systematic exploration of the five-dimensional parameter space of the model, which corroborates the previous indicative results. We identify the small regions of parameter space which satisfy both constraints and show that the cosmologically acceptable region should be almost fully testable at LEPII and/or SuperKamiokande.

The minimal $S U(5)$ supergravity model consists of the Standard Model fields (with two Higgs doublets) and their superpartners, plus the heavy GUT fields in the form of colored Higgs triplet fields $(H, \bar{H})$ and gauge and Higgs bosons in the $\mathbf{2 4}$ representation of $S U(5)$. Universal soft-supersymmetry breaking at the scale $M_{U}$ is described by three parameters: $m_{1 / 2}, m_{0}, A$. The $S U(5)$ gauge symmetry entails gauge coupling $\left(\alpha_{1}=\alpha_{2}=\alpha_{3}=\alpha_{5}\right.$ at $\left.M_{U}\right)$ and Yukawa coupling $\left(\lambda_{b}=\lambda_{\tau}\right.$ at $\left.M_{U}\right)$ unification. In our calculation all these parameters are run from $M_{U}$ down to low energies $\left(M_{Z}\right)$ as prescribed by the relevant renormalization group equations. Finally, at the scale $M_{Z}$ the full one-loop effective potential is minimized and the one-loop corrected Higgs boson masses are obtained. The final parameter list is: $\tan \beta, m_{t}, m_{1 / 2}, \xi_{0} \equiv m_{0} / m_{1 / 2}, \xi_{A} \equiv A / m_{1 / 2}$, and the sign of the Higgs mixing term $(\mu)$. This set of parameters should be constrasted with the 21dimensional parameter space of the usual MSSM. For recent analyses of this type, see Refs. [12, 13, 114, 15, 16]. 


\section{Standard Constraints}

We impose the following set of 'standard constraints' on the parameter space of the model (see Ref. [16] for a detailed discussion):

(i) one-loop radiative electroweak symmetry breaking;

(ii) perturbative unification (which implies $m_{t} \lesssim 190 \mathrm{GeV}$ and $\tan \beta \lesssim 50$ );

(iii) $m_{\tilde{q}, \tilde{l}}^{2}>0$;

(iv) a neutral and colorless LSP $\left(i . e ., \tilde{\nu}\right.$ or $\left.\chi \equiv \chi_{1}^{0}\right)$;

(v) experimental bounds on $m_{\chi_{1}^{+}}, m_{\tilde{l}}, m_{\tilde{g}}, m_{\tilde{q}}, m_{t}, \Gamma_{i n v}$;

(vi) $m_{\tilde{q}}^{a v e}, m_{\tilde{g}}<1 \mathrm{TeV}$, motivated by naturalness considerations, or demanding testability of the model at the SSC;

(vii) $\lambda_{b}\left(M_{U}\right)=\lambda_{\tau}\left(M_{U}\right)$, which in practice we use to predict $m_{b}\left(m_{b}\right)$ for a given set of $m_{t}$, $\tan \beta$, and $\alpha_{3}$ values.

We have explored the following hypercube of the parameter space: $\mu>0, \mu<0$, $\tan \beta=2-10(2), m_{t}=100-160(5), \xi_{0}=0-10(1), \xi_{A}=-\xi_{0}, 0,+\xi_{0}$, and $m_{1 / 2}=$ $50-300$ (6), where the numbers in parenthesis represent the size of the step taken in that particular direction. (Points outside these ranges have little (a posteriori) likelihood of being acceptable.) Of these $92,235 \times 2=184,470$ points, $\approx 25 \%$ passed all the standard constraints. In what follows we present our results as a collection of scatter plots, where a given pair of observables is plotted for each allowed point in the parameter space.

\section{Proton Decay}

In unified supersymmetric theories only the dimension-five-mediated proton decay operators are constraining. In calculating the proton lifetime we consider the typically dominant decay modes $p \rightarrow \bar{\nu}_{\mu, \tau} K^{+}$and neglect all other possible modes. Schematically the lifetime is given by []

$$
\tau_{p} \equiv \tau\left(p \rightarrow \bar{\nu}_{\mu, \tau} K^{+}\right) \sim\left|M_{H} \sin 2 \beta \frac{1}{f} \frac{1}{1+y^{t K}}\right|^{2} .
$$

Here $M_{H}$ is the mass of the exchanged GUT Higgs triplet which on perturbative grounds is assumed to be bounded above by $M_{H}<3 M_{U}$ [6.9.10]; $\sin 2 \beta=2 \tan \beta /\left(1+\tan ^{2} \beta\right)$, thus $\tau_{p}$ 'likes' small $\tan \beta$ (we find that only $\tan \beta \leq 6$ is allowed); $1+y^{t K}$ represents the calculable ratio of the third- to the second-generation contributions to the dressing one-loop diagrams. An unkown phase appears in this ratio and we always consider the 
weakest possible case of destructive interference. Finally $f$ represents the sparticle-massdependent dressing one-loop function which decreases asymptotically with large sparticle masses.

In Fig. 1 (top row) we show a scatter plot of $\left(\tau_{p}, m_{\tilde{g}}\right)$. The various 'branches' correspond to fixed values of $\xi_{0}$. Note that for $\xi_{0}<3, \tau_{p}<\tau_{p}^{e x p}=1 \times 10^{32}$ y (at 90\% C.L. [17]). Also, for a given value of $\xi_{0}$, there is a corresponding allowed interval in $m_{\tilde{g}}$. The lower end of this interval is determined by the fact that $\tau_{p} \propto 1 / f^{2}$, and $f \approx m_{\chi_{1}^{+}} / m_{\tilde{q}}^{2} \propto 1 / m_{\tilde{g}}\left(c+\xi_{0}^{2}\right)$, in the proton-decay-favored limit of $\mu \gg M_{W}$; thus $m_{\tilde{g}}\left(c+\xi_{0}^{2}\right)>$ constant. The upper end of the interval follows from our requirement $m_{\tilde{q}}\left(\propto m_{\tilde{g}} \sqrt{c+\xi_{0}^{2}}\right)<1 \mathrm{TeV}$. Statistically speaking, the proton decay cut is quite severe, allowing only about $\sim 1 / 10$ of the points which passed all the standard constraints, independently of the sign of $\mu$.

Note that if we take $M_{H}=M_{U}$ (instead of $\left.M_{H}=3 M_{U}\right)$, then $\tau_{p} \rightarrow \frac{1}{9} \tau_{p}$ and all points in Fig. 1 would become excluded. To obtain a rigorous lower bound on $M_{H}$, we would need to explore the lowest possible allowed values of $\tan \beta$ (in Fig. 1, $\tan \beta \geq 2$ ). Roughly, since the dominant $\tan \beta$ dependence of $\tau_{p}$ is through the explicit $\sin 2 \beta$ factor, the upper bound $\tau_{p} \lesssim 8 \times 10^{32}$ y for $\tan \beta=2$, would become $\tau_{p} \lesssim 1 \times 10^{33}$ y for $\tan \beta=1$. Therefore, the current experimental lower bound on $\tau_{p}$ would imply $M_{H} \gtrsim M_{U}$. Note also that SuperKamiokande $\left(\tau_{p}^{\text {exp }} \approx 2 \times 10^{33}\right.$ y) should be able to probe the whole allowed range of $\tau_{p}$ values.

The actual value of $\alpha_{3}\left(M_{Z}\right)$ used in the calculations $\left(\alpha_{3}=0.120\right.$ in all figures $)$ has a non-negligible effect of some of the final results, mostly due to its effect on the value of $M_{H}=3 M_{U}$ : larger values of $\alpha_{3}$ increase $M_{U}$ and therefore $\tau_{p}$, and thus open up the parameter space, and viceversa. For example, for $\alpha_{3}=0.113(0.120)$ we get $m_{\tilde{g}} \lesssim$ $550(800) \mathrm{GeV}, \xi_{0} \geq 5(3)$, and $\tau_{p} \lesssim 4(8) \times 10^{32} \mathrm{y}$.

\section{Cosmology}

We assume that the lightest neutralino is a stable particle, as expected in the minimal, $R$-parity conserving model. The current cosmological observations of $\Omega_{0} \lesssim 1$ [18] and/or the inflation prediction $\Omega_{0}=1$ [19], lead us to impose the constraint $\Omega_{\chi} h_{0}^{2} \leq 1$. In Fig. 2 we show the calculated valuest of $\Omega_{\chi} h_{0}^{2}$ versus $m_{\chi}$, which show a noticeable dip at

1 For a detailed discussion of the methods used to compute $\Omega_{\chi} h_{0}^{2}$ see Ref. [20]. For computations of $\Omega_{\chi} h_{0}^{2}$ in supergravity models with radiative electroweak symmetry breaking see [13, 14, 21]. 
$m_{\chi} \approx \frac{1}{2} M_{Z}$ due to $s$-channel $Z$-pole annihilation. Only $\sim 1 / 6$ of the points have $\Omega_{\chi} h_{0}^{2} \leq 1$. This result is not unexpected since proton decay is suppressed by heavy sparticle masses, whereas $\Omega_{\chi} h_{0}^{2}$ is enhanced. Therefore, a delicate balance needs to be attained to satisfy both constraints simultaneously. The subset of cosmologically allowed points does not change the range of possible $\tau_{p}$ values (see Fig. 1 bottom row), although it depletes the constant- $\xi_{0}$ 'branches'.

In Fig. 3 we show the calculated values of $\mu$ with and without the cosmological constraint. As expected, values of $\mu$ giving nearly pure gaugino $\chi$-compositions (above the $|\mu|=M_{2}=0.3 m_{\tilde{g}}$ line) are cosmologically disfavored since in this limit the $\chi \chi Z$ and $\chi \chi h$ couplings are highly suppressed. Exceptions to this rule can be traced to $m_{\tilde{g}}$ values for which $m_{\chi} \approx \frac{1}{2} M_{Z}, \frac{1}{2} m_{h}$ when the annihilation cross section is enhanced by $s$-channel poles. Also, since $|\mu|$ grows with $m_{t}$, larger values of $m_{t}$ tend to be disfavored as well. The results are quite similar for the $\alpha_{3}=0.113$ case.

\section{Particle Mass Correlations}

Since Fig. 3 shows that proton decay generally requires $\mu \gg M_{W}$ (and to a somewhat lesser extent also $\mu \gg M_{2}$ ), the lightest chargino will have mass $m_{\chi_{1}^{+}} \approx M_{2} \approx 0.3 m_{\tilde{g}}$, whereas the two lightest neutralinos will have masses $m_{\chi} \approx M_{1} \approx \frac{1}{2} M_{2}$ and $m_{\chi_{2}^{0}} \approx M_{2}$ [9]. Thus, within some approximation we expect

$$
m_{\chi} \approx \frac{1}{2} m_{\chi_{2}^{0}}, \quad m_{\chi_{2}^{0}} \approx m_{\chi_{1}^{+}}, \quad m_{\chi} \approx 0.15 m_{\tilde{g}}
$$

The calculated values of these masses are shown in Fig. 4 (without imposing the cosmological constraint). The approximate mass relations are quite accurate for $\mu>0$, but more qualitative for $\mu<0$. Inclusion of the cosmological constraint basically just depletes the point density without affecting significantly the range of particle masses. The value of $\alpha_{3}$ does not affect these mass relations either, although the particle mass ranges do change

$$
m_{\chi}<85(115) \mathrm{GeV}, \quad m_{\chi_{2}^{0}, \chi_{1}^{+}}<165(225) \mathrm{GeV}, \quad \text { for } \alpha_{3}=0.113(0.120)
$$

We also find that the one-loop corrected lightest Higgs boson mass $\left(m_{h}\right)$ is bounded above by

$$
m_{h} \lesssim 110 \mathrm{GeV}
$$


independently of the sign of $\mu$, the value of $\alpha_{3}$, or the cosmological constraint. In Fig. 5 we plot $m_{h}$ versus $m_{\chi_{1}^{+}}$which shows an experimentally interesting correlation if the cosmological constraints are imposed (bottom row). Indeed,

$$
m_{h} \gtrsim 75(80) \mathrm{GeV} \Rightarrow m_{\chi_{1}^{+}} \lesssim 90(110) \mathrm{GeV},
$$

for $\alpha_{3}=0.113(0.120)$. Moreover, the correlations among the lightest chargino and neutralino masses in Eq. (5.1) imply analogous results for $\left(m_{h}, m_{\chi_{2}^{0}}\right)$ and $\left(m_{h}, m_{\chi}\right)$,

$$
m_{h} \gtrsim 80 \mathrm{GeV} \Rightarrow m_{\chi_{2}^{0}} \lesssim 90(110) \mathrm{GeV}, \quad m_{\chi} \lesssim 48(60) \mathrm{GeV},
$$

for $\alpha_{3}=0.113(0.120)$. These correlations can be understood in the following way: since we find that $m_{A} \gg M_{Z}$, then $m_{h} \approx|\cos 2 \beta| M_{Z}+$ (rad. corr.). In the situation we consider here, we have determined that all of the allowed points for $m_{\tilde{g}}>400 \mathrm{GeV}$ correspond to $\tan \beta=2$. This implies that the tree-level contribution to $m_{h}$ is $\approx 55 \mathrm{GeV}$. that the cosmology cut restricts $m_{t}<130(140) \mathrm{GeV}$ for $\mu>0(\mu<0)$ in this range of $m_{\tilde{g}}$. Therefore, the radiative correction contribution to $m_{h}^{2}\left(\propto m_{t}^{4}\right)$ will be modest in this range of $m_{\tilde{g}}$. This explains the depletion of points for $m_{h} \gtrsim 80 \mathrm{GeV}$ in Fig. 5 and leads to the mass relationships in Eqs. (5.4) and (5.5).

Recent studies of Higgs searches relevant to LEPII have shown that large regions of the parameter space which determine $m_{h}$, including radiative corrections, can be explored [22,23]. These studies make simplifying assumptions regarding the many parameters at low energy, as well as choosing fixed values of $m_{t}$. Nonetheless, we expect a certain level of quantitative agreement with these generic analyses. Important conclusions tend to be unanimous; if $\tan \beta \lesssim 5$, then values of $m_{h}$ up to $m_{h} \approx 80 \mathrm{GeV}$ can be explored (see e.g., Figs. 2a,8a in [22]). This is precisely the constraint on $\tan \beta$ that is realized in our analysis due to the stringent proton decay cuts. As we have discussed, the regions in parameter space where $m_{h} \gtrsim 80 \mathrm{GeV}$ result in the constraint $m_{\chi_{1}^{+}} \lesssim 90(110) \mathrm{GeV}$ for $\alpha_{3}=0.113(0.120)$, and both signs of $\mu$. Early model-dependent analyses of $e^{+} e^{-} \rightarrow \widetilde{W} l \tilde{\nu}_{l}$ indicate that $m_{\widetilde{W}} \lesssim 100 \mathrm{GeV}$ could be explored at LEPII [24]. A more careful upper limit would require a detailed calculation, but these results are encouraging nonetheless. Therefore, if LEPII does not see the lightest Higgs, it has a good chance of seeing the lightest chargino instead, or viceversa.

2 Note that Fig. 5 cannot be used to establish a lower bound on $m_{h}$ since values of $1<\tan \beta<2$ have not been considered. This is unlike the case for the quoted upper bound in Eq. (5.3). 


\section{Conclusions}

The most direct and pervasive evidence for unified models would be the observation of nucleon decay. In fact, this kind of test of a unified model has the very appealing property of involving the physics of both low-mass and high-mass particles in the theory, and as such should be able to discriminate among the various competing unified models at hand. In the specific case of the minimal $S U(5)$ supergravity model, we have shown that under sensible assumptions, the range of proton lifetimes still to be probed by the next round of proton decay experiments is finite and completely accessible. Besides the various uncertainties on the measured 'constants' which enter the proton lifetime formula, two assumptions are key to the results presented in this paper: (i) the upper bound on the Higgs triplet mass $M_{H}<3 M_{U}$, and (ii) the assumed upper bound on the squark and gluino masses of $1 \mathrm{TeV}$. Relaxing any of these assumptions can suppress the proton lifetime to acceptable values. However, we believe that if these quite sensible assumptions had to be relaxed to ensure compatibility with experimental requirements, then much of the motivation to consider this model would fade away and in effect the model would become 'sociologically' excluded. Another important variable in this study is the value of $\alpha_{3}$, larger values of which tend to open up the parameter space. We have considered the case of $\alpha_{3}=0.113$ and 0.120 . A better defined value for this quantity will go a long way in nailing the specific predictions of the model.

The recent precise observation by the COBE satellite of minute anisotropies in the cosmic microwave background radiation [25] is a dramatic reminder that precision cosmology has come to age, and that a unified model should be judged seriously by its consistency with cosmological constraints. In the present model we have shown that a suitable value for the cosmological relic density of the lightest neutralino is another powerful constraint on the model parameters. In effect it reduces the size of the allowed parameter space by a factor of 6 .

The proton decay cut singles out a small region of the parameter space where a number of fairly accurate mass correlations and mass bounds exists. We find for the oneloop corrected lightest Higgs boson mass $m_{h} \lesssim 110 \mathrm{GeV}$, independently of the sign of $\mu$, the value of $\alpha_{3}$, or the cosmological constraint. Since $|\mu| \gg M_{W}$ holds for most of the

allowed parameter space, it also follows that $m_{\chi} \approx \frac{1}{2} m_{\chi_{2}^{0}} \approx 0.15 m_{\tilde{g}}$ and $m_{\chi_{2}^{0}} \approx m_{\chi_{1}^{+}}$. The imposed upper bound $m_{\tilde{q}}<1 \mathrm{TeV}$ cuts off the value of the gluino mass for a given $\xi_{0}$ value and gives upper bounds on the light particle masses: $m_{\chi}<85(115) \mathrm{GeV}$ and 
$m_{\chi_{2}^{0}, \chi_{1}^{+}}<165(225) \mathrm{GeV}$ for $\alpha_{3}=0.113(0.120)$. The effect of the cosmological cut on these predictions is negligible. However, this cut does remove a large portion of the allowed points and has a dramatic effect on the correlation between $m_{h}$ and $m_{\chi_{1}^{+}}$(or $m_{\chi_{2}^{0}}, m_{\chi}$ since they are all related), such that at least one of these particles is quite likely to be observable at LEPII. To recapitulate, of the 184,470 points in parameter space which we examined, only $\sim 1 / 240$ satisfy the standard constraints, the proton decay bound, and the cosmological requirement, leaving only a rather restricted set of points to be put to experimental test. In sum, it should not be long before we could start discriminating among the various supersymmetric unified models.

Acknowledgments: This work has been supported in part by DOE grant DE-FG05-91ER-40633. The work of J.L. has been supported in part by an ICSC-World Laboratory Scholarship and in part by an SSC Fellowship. The work of D.V.N. has been supported in part by a grant from Conoco Inc. We would like to thank the HARC Supercomputer Center for the use of their NEC SX-3 supercomputer. 


\section{References}

[1] A. Chamseddine, R. Arnowitt, and P. Nath, Phys. Rev. Lett. 49 (1982) 970; For reviews see: R. Arnowitt and P. Nath, Applied N=1 Supergravity (World Scientific, Singapore 1983); H. P. Nilles, Phys. Rep. 110 (1984) 1.

[2] J. Ellis, S. Kelley and D. V. Nanopoulos, Phys. Lett. B 249 (1990) 441, Phys. Lett. B 260 (1991) 131; P. Langacker and M.-X. Luo, Phys. Rev. D 44 (1991) 817; U. Amaldi, W. de Boer, and H. Fürstenau, Phys. Lett. B 260 (1991) 447; F. Anselmo, L. Cifarelli, A. Peterman, and A. Zichichi, Nuovo Cim. 104A (1991) 1817.

[3] J. Ellis, S. Kelley, and D. V. Nanopoulos, Nucl. Phys. B 373 (1992) 55 and Phys. Lett. B 287 (1992) 95; R. Barbieri and L. Hall, Phys. Rev. Lett. 68 (1992) 752; F. Anselmo, L. Cifarelli, A. Peterman, and A. Zichichi, Nuovo Cim. 105A (1992) 581; J. Hisano, H. Murayama, and T. Yanagida, Phys. Rev. Lett. 69 (1992) 1014.

[4] S. Weinberg, Phys. Rev. D 26 (1982) 287; N. Sakai and T. Yanagida, Nucl. Phys. B 197 (1982) 533.

[5] J. Ellis, D. V. Nanopoulos, and S. Rudaz, Nucl. Phys. B 202 (1982) 43; B. Campbell, J. Ellis, and D. V. Nanopoulos, Phys. Lett. B 141 (1984) 229.

[6] K. Enqvist, A. Masiero, and D. V. Nanopoulos, Phys. Lett. B 156 (1985) 209.

[7] P. Nath, A. Chamseddine, and R. Arnowitt, Phys. Rev. D 32 (1985) 2348; P. Nath and R. Arnowitt, Phys. Rev. D 38 (1988) 1479.

[8] M. Matsumoto, J. Arafune, H. Tanaka, and K. Shiraishi, University of Tokyo preprint ICRR-267-92-5 (April 1992).

[9] R. Arnowitt and P. Nath, Phys. Rev. Lett. 69 (1992) 725; P. Nath and R. Arnowitt, Phys. Lett. B 287 (1992) 89 and NUB-TH-3048-92 and CTP-TAMU-27-92.

[10] J. Hisano, H. Murayama, and T. Yanagida, Tohoku University preprint TU-400 (July 1992).

[11] J. L. Lopez, D. V. Nanopoulos, and A. Zichichi, Texas A \& M University preprint CTP-TAMU-49/92 and CERN-TH.6554/92.

[12] G. Gamberini, G. Ridolfi, and F. Zwirner, Nucl. Phys. B 331 (1990) 331.

[13] J. Ellis and F. Zwirner, Nucl. Phys. B 338 (1990) 317.

[14] S. Kelley, J. L. Lopez, D. V. Nanopoulos, H. Pois, and K. Yuan, Phys. Lett. B 273 (1991) 423.

[15] K. Inoue, M. Kawasaki, M. Yamaguchi, and T. Yanagida, Phys. Rev. D 45 (1992) 328; G. G. Ross and R. G. Roberts, Nucl. Phys. B 377 (1992) 571; M. Drees and M. M. Nojiri, Phys. Rev. D 45 (1992) 2482.

[16] S. Kelley, J. L. Lopez, D. V. Nanopoulos, H. Pois, and K. Yuan, Texas A \& M University preprint CTP-TAMU-16/92 and CERN-TH.6498/92.

[17] Particle Data Group, Phys. Rev. D 45 (1992) S1.

[18] See e.g., E. Kolb and M. Turner, The Early Universe (Addison-Wesley, 1990). 
[19] For recent reviews see e.g., K. Olive, Phys. Rep. 190 (1990) 307; D. Goldwirth and T. Piran, Phys. Rep. 214 (1992) 223.

[20] J. L. Lopez, D. V. Nanopoulos, and K. Yuan, Nucl. Phys. B 370 (1992) 445.

[21] M. Kawasaki and S. Mizuta, Phys. Rev. D 46 (1992) 1634; S. Kelley, J. L. Lopez, D. V. Nanopoulos, H. Pois, and K. Yuan, Texas A \& M University preprint CTPTAMU-56/92 and CERN-TH.6584/92; M. Drees and M. M. Nojiri, SLAC preprint SLAC-PUB-5860 (July 1992).

[22] Z. Kunszt and F. Zwirner, CERN-TH.6150/91, ETH-TH/91-7.

[23] V. Barger, K. Cheung, A. L. Stange, and R. Phillips, MAD-PH-704, June 1992; H. Baer, C. Kao, M. Bisset, X. Tata, and D. Dicus, FSU-HEP-920724. For a recent review see J. Gunion, UCD-92-20.

[24] M. Chen, C. Dionisi, M. Martinez, and X. Tata, Phys. Rep. 159 (1988) 203.

[25] G. Smoot, et. al., COBE preprint (1992). 


\section{Figure Captions}

Fig. 1. Scatter plot of the proton lifetime $\tau_{p} \equiv \tau\left(p \rightarrow \bar{\nu}_{\mu, \tau} K^{+}\right)$versus the gluino mass for the hypercube of the parameter space explored. The current experimental lower bound is $\tau_{p}^{\text {exp }}=1 \times 10^{32} \mathrm{y}$. The various 'branches' correspond to fixed values of $\xi_{0}$ as indicated (the labelling applies to all four windows). The bottom row includes the cosmological constraint. The upper bound on $m_{\tilde{g}}$ follows from the requirement $m_{\tilde{q}}<1 \mathrm{TeV}$.

Fig. 2. The calculated neutralino relic density $\Omega_{\chi} h_{0}^{2}$ versus the neutralino mass. Note the effect of the $s$-channel $Z$-pole at $m_{\chi} \approx \frac{1}{2} M_{Z}$. Only about $1 / 6$ of the points have $\Omega_{\chi} h_{0}^{2} \leq 1$.

Fig. 3. The calculated value of $\mu$ versus $m_{\tilde{g}}$. The cosmological constraint is enforced in the bottom row. Above the solid lines (labelled $\widetilde{B}$ ) the neutralino is a nearly pure bino eigenstate, above the line $|\mu|=M_{2}=0.3 m_{\tilde{g}}$ it is mostly a gaugino eigenstate. The small regions in the bottom row plots which extend up into the pure bino regions correspond to $m_{\chi}$ masses near $\frac{1}{2} m_{Z}$ and $\frac{1}{2} m_{h}$.

Fig. 4. The lightest neutralino mass $\left(m_{\chi}\right)$ versus the gluino mass (top row) and the second-to-lightest neutralino mass $\left(m_{\chi_{2}^{0}}\right)$ versus the lightest chargino mass $\left(m_{\chi_{1}^{+}}\right)$ (bottom row). These plots show the anticipated approximate mass correlations due to the proton decay constraint, $m_{\chi} \approx 0.15 m_{\tilde{g}}, m_{\chi_{2}^{0}} \approx m_{\chi_{1}^{+}} \approx 2 m_{\chi}$. The cosmological constraint (not enforced in this figure) simply depletes the point density, without affecting the range of the particle masses.

Fig. 5. The one-loop corrected lightest Higgs boson mass $\left(m_{h}\right)$ versus the lightest chargino mass. If the cosmological constraint is enforced (bottom row), then for $m_{h} \gtrsim 80 \mathrm{GeV}$ the lightest chargino is below $110 \mathrm{GeV}$. Thus, at least one of these particles is likely to be discovered at LEPII. A significant number of the allowed points correspond to $m_{\chi}$ masses near $\frac{1}{2} M_{Z}$ and $\frac{1}{2} m_{h}$. 